\title{
Cathodoluminescence of polished carbonado
}

\author{
H.J. Milledge ${ }^{1}$, P.A.Woods ${ }^{1}$, A.D. Beard ${ }^{1}$, D. Shelkov ${ }^{2} \&$ B. Willis ${ }^{3}$
}

1)Research School of Geological \& Geophysical Sciences, Birkbeck \& University College London, Gower St., London WCIE 6BT, United Kingdom.

2)Planetary Sciences Research Institute, The Open University, Milton Keynes MK7 6AA, United Kingdom

3)Research School of Earth Sciences, The Australian National University, GPO Box 4, Canberra ACT 2601, Australia.

Previous investigations of groups of carbonados from Ubangui and from Brazil have been described by Shelkov et al. (1997). In particular, carbon and nitrogen isotope data were similar for specimens from both sources, and similar to values found for polycrystalline diamond from Jwaneng and Orapa, suggesting that a Mantle origin was more likely than the crustal origin proposed on the basis of inclusions such as florencite frequently found in carbonado. This view was reinforced by infrared spectra of Type IaA obtained from one Brazilian specimen containing large crystallites.

These carbonados had been fractured in order to identify inclusions without contamination during a polishing process, and the presence of veneers rich in rare-earth components of the type previously reported was confirmed for both Brazilian and Ubangui specimens. Cathodoluminescence (CL) examinations of both natural and fractured surfaces were made for these and for some 60 other carbonados, some of unknown origin. External surfaces were predominantly red luminescent, while internal surfaces were more variable, but no fractured microcrystals were observed.

An attempt was made to polish pieces of these specimens in order to see whether the smooth crust observed on the exterior of some of them, giving them a "flame-polished" appearance, was pore-free, in which case geochemical data on the inclusions might be considered as related to the diamond genesis rather than to have been introduced or altered after emplacement, and to give some idea of the textural variations to be found, which were considerable, but the CL of these polished surfaces was not examined.

However, a small Brazilian carbonado was polished at the Australian National University Research School of Earth Sciences in 1996 to see whether a reasonably good finish could be obtained using techniques in use there, so that any large inclusions encountered could be probed in the usual way. In the course of probe measurements at University College London, backscattered images showed that the specimen was essentially pure carbon, though containing many holes from which inclusions may have been removed, and the CL was checked to see whether the colour would differ from the predominantly reddish CL usually seen on external surfaces.

This revealed an astonishing array of zoned microdiamonds up to $\sim 50$ microns in diameter, embedded in a featureless, apparently fine-grained, diamond matrix. CL spectra were subsequently obtained, at very high spatial resolution at liquid nitrogen temperatures in our SEM, from cores and individual zones of some of these microdiamonds, and showed that the $574 \mathrm{~nm}$ system was much stronger than any other system and was extended by a broad band without any observable zero-phonon component past 700nm, thus accounting for the general red CL of carbonado. The $574 \mathrm{~nm}$ system was dominant even in the 
internal zones of these microdiamonds, and is ascribed to a (nitrogen + vacancy) centre usually, but not always (as for example in some synthetic diamond) associated with radiation damage.

As far as could be seen, there were no discrete haloes of the type seen on the external surfaces of many diamonds, but the specimens of the Ubangui carbons polished previously were now examined in CL. These showed occasional microdiamonds of a size similar to those seen in the Brazilian specimen, but otherwise a featureless carbon surface containing many cracks. a number of which were decorated by radioactive haloes characteristic of the Uranium decay chain (Mendelssohn et. al. 1979). The large number of these haloes could well account for the high He content found for carbonado, comparable to that measured for the green skins of alpha-damaged diamonds at the Open University, and attributed to the action of ground water after emplacement.

Recently Ozima and Tatsumoto (1997), on the basis of a study of the U-Th-Pb systematics of ten carbonados from the Central African Republic, have supported the suggestion that carbonado represents radiation-induced crystallisation, but the very extensive halo distribution associated with cracks in the Ubangui carbonados studied here makes it seem likely that uranium was only involved after emplacement in such specimens.

A large number of fractured carbonados were re-examined in CL, but no microdiamond sections were encountered, indicating that intergranular fracture is more likely than intragranular fracture.

\section{References}

Mendelssohn, M.J. \& Milledge, H.J., Nave, E, Vance, E.R. \& Woods, P.A. (1979).Diamond Research. 35-42 "Internal Radioactive Haloes in Diamond".

Ozima,M. \& Tatsumoto,M. (1997) Geocchem. et Cosmochim. Acta 61, 369-376. "Radiation-induced diamond crystallisation: Origin of carbonados and its implications on meteorite nanodiamonds."

Shelkov, D., Verkhovsky,A.B., Milledge, H.J. and Pillinger, C.T. (1997) Russ. Geol \& Geophys. 38, No.2, 332-340. "Carbonado: a comparison between Brazilian and Ubangui sources with other forms of microcrystalline diamond based on carbon and nitrogen isotopes." 